\title{
Surgery for congenital diseases of the aorta
}

\author{
Duke Cameron, MD
}

\begin{abstract}
Congenital diseases of the aorta tend to be obstructive when they present early in life, and aneurysmal when they present later in life. The latter group also tends to be associated with connective tissue disorders and with repaired conotruncal lesions. The indications for intervention in the aneurysm group are still in evolution but are clearly age- and lesion-dependant. Disorders such as Loeys-Dietz syndrome and Turner syndrome may deserve aggressive prophylactic surgery, as well as Marfan syndrome to a lesser extent. The natural history of the dilated aorta after repair of congenital heart lesions is probably more benign than de novo aneurysms and therefore should be treated conservatively. (J Thorac Cardiovasc Surg 2015;149:S14-7)
\end{abstract}

Congenital diseases of the aorta can be divided into 2 major categories: those that present problems early in life and those with later manifestations. The early lesions are usually obstructive in nature: they include coarctation of the aorta, hypoplastic left heart syndrome, and vascular rings, and tend to involve the arch of the aorta. The later-onset congenital aortic problems are chiefly aneurysmal and involve predominantly the ascending aorta. ${ }^{1}$ They include the bicuspid aortic valve (BAV), Marfan syndrome, Loeys-Dietz syndrome, and Turner syndrome. Apart from Turner syndrome, these aneurysmal diseases of the aorta are addressed elsewhere in this symposium, and so will not be included in this discussion. Rather, I will focus principally on the patients who have undergone surgery for various congenital cardiac lesions, and then present later with aortic complications, usually aneurysmal in nature. These are particularly challenging problems because of the paucity of data on which to base management decisions. These aneurysms typically follow surgery for conotruncal lesions such as tetralogy of Fallot (TOF), persistent truncus arteriosus communis, and double-outlet right ventricle (DORV), but also after the Norwood procedure, the arterial switch, and the Ross procedure.

\section{COARCTATION OF THE AORTA}

Most of the current techniques for repair of coarctation of the aorta and complete interruption of the aortic arch-obstructions that occur most frequently just distal to the left subclavian artery-have been in use

\footnotetext{
From the Division of Cardiac Surgery, The Johns Hopkins Medical Institutions, Baltimore, Md.

Disclosures: Author has nothing to disclose with regard to commercial support.

Read at The American Association for Thoracic Surgery Aortic Symposium, New York, New York, April 24-25, 2014.

Received for publication Sept 29, 2014; accepted for publication Oct 19, 2014.

Address for reprints: Duke Cameron, MD, Division of Cardiac Surgery, The Johns Hopkins Hospital, Zayed 7107, 1800 Orleans St, Baltimore, MD 21287 (E-mail: dcameron@jhmi.edu).

$0022-5223 / \$ 36.00$

Copyright (c) 2015 by The American Association for Thoracic Surgery

http://dx.doi.org/10.1016/j.jtcvs.2014.10.084
}

for the past 30 years, and generally involve complete resection of the involved segment followed by primary anastomosis of the proximal and distal aorta. Primary repair allows the aorta to grow appropriately and parallel to somatic growth. If subsequent obstruction occurs, this can often be dealt with successfully using catheter-based interventions.

Some of the earlier approaches to aortic arch obstruction involved patch augmentation of the narrowed segment or interposition grafts using prosthetic materials such as Dacron, often with incomplete resection of the narrowed segment and its pathologic tissue. Repairs involving Dacron patches have not only resulted in occasional residual obstruction but also an alarming incidence of late aneurysm and/or dissection of the aorta. In a recent single-center study from the University of Wisconsin, $10 \%$ of patients with patch repair had aneurysms at 10 years, and more than $50 \%$ had aneurysms at 25 years; $14 \%$ of these experienced rupture within 20 years, and half of those patients died. ${ }^{2}$ This suggests that close surveillance of patients with Dacron patch aortoplasty is mandatory, and early reintervention is justified.

\section{AORTIC DILATATION FOLLOWING REPAIR OF CONOTRUNCAL LESIONS}

With improvement in the results of initial surgery for congenital heart disease and greater repair and patient longevity, there are now more adults living with congenital heart disease than children. ${ }^{3}$ Among these are patients who have undergone repair of conotruncal defects (ie, TOF, DORV, and truncus arteriosus), the arterial switch procedure for complete transposition of the great arteries (TGA), the Norwood procedure for hypoplastic left heart syndrome (HLHS), and the Ross procedure for aortic valve disease. In all of these conditions, the aorta tends to dilate, posing a theoretical risk of late rupture and/or dissection, an increased likelihood of aortic valve incompetence, and the possibility of compression of adjacent structures. The sheer size of the aorta in some patients with the Fontan procedure, in which pulmonary blood 

Abbreviations and Acronyms
$\mathrm{BAV}=$ bicuspid aortic valve
DORV $=$ double-outlet right ventricle
HLHS $=$ hypoplastic left heart syndrome
TGA $=$ transposition of the great arteries
$\mathrm{TOF}=$ tetralogy of Fallot

flow is provided passively from the systemic venous system, poses a particular problem as the dilated aorta may compress the pulmonary arteries and obstruct systemic venous return.

From an embryologic perspective, we can view all these congenital anomalies as arising from various perturbations of the partitioning of the outflow tracts of the heart. The outflow tract is initially a single tube, with the right ventricular conus leading into the truncus arteriosus. This outflow tract is normally divided by the combined aorticopulmonary and conotruncal septa into roughly equal channels, providing pulmonary flow from the right ventricle and aortic flow from the left ventricle (Figure 1).

Perhaps the most easily understood example of unbalanced division of the conotruncus is TOF. In this condition, the septum between the right ventricular outflow to the pulmonary artery and the left ventricular outflow to the aorta is shifted too far to the right, leading to obstruction of the right ventricular outflow tract and resulting in various degrees of pulmonary stenosis or pulmonary atresia. The malposition of the septum also results in a large aorta and prevents the infundibular portion of the conotruncal/aortopulmonary septum from connecting with the muscular/membranous interventricular septum inferiorly, leading to the characteristic malalignment ventricular septal defect.

If the conotruncal/aortopulmonary septum is shifted too far to the left, it severely restricts aortic outflow, with devastating effects on the development of the left ventricle, ascending aorta, and aortic arch, leading to HLHS, interruption of the aortic arch, and various forms of coarctation. Another type of conotruncal abnormality is TGA, in which the septum fails to undergo its usual spiral rotation during development, resulting in a right ventricle that empties into the aorta and a left ventricle with outflow into the pulmonary artery. Complete TGA is uniformly fatal without intervention unless a mixing lesion is present, such as a patent ductus arteriosus or an atrial or ventricular septal defect.

Regardless of the nature of the septation abnormality, individuals with these conotruncal lesions have an abnormally weak and often enlarged aorta from infancy. Histologic examination reveals that there is some fragmentation and loss of the elastic lamellae present already at birth, which also progresses with age. ${ }^{4}$ The degree of disruption of the normal architecture of the aortic wall correlates roughly with the degree of aortic dilatation. Most of the data available to us come from patients with the more common conotruncal lesions, chiefly TOF, because these patients historically had the earliest repairs and have lived the longest. All TOF patients have a dilated aorta at birth, and regardless of their age at surgery and the type of intervention, the aorta continues to enlarge throughout life. Although these aortas remain large even after early intracardiac repair and continue to grow throughout life, there are only rare instances in which rupture or dissection have been reported. In the dozen or so reported cases of surgical repair, aortic replacement was generally required more than 20 years after initial repair of TOF and usually for moderate or severe aortic incompetence rather than rupture or dissection. Older age at repair, male gender, and pulmonary atresia appear to be risk factors for aortic aneurysm in TOF patients. ${ }^{5}$ But there are too few cases to generate reliable guidelines for elective intervention for the dilated aorta in TOF.

\section{Aortic Dilatation After the Arterial Switch, Norwood, and Ross Procedures}

The arterial switch operation-one of the greatest success stories in the treatment of congenital heart disease - has given near-normal life expectancy and quality of life to patients with complete TGA, rescuing them from dismal and uniformly fatal natural history. The arterial switch operation is technically demanding but enjoys a high level of success, both early and late after repair. Although it is frequently called an anatomic correction, it is actually not: the great arteries are switched so that the aorta is connected to the left ventricle and the pulmonary artery to the right ventricle and the coronary arteries are transferred, but the aortic and pulmonary roots (including their respective semilunar valves), remain unswitched. In addition to the intrinsic differences between the 2 semilunar valves and the sinus tissue that encircles them, the root of each great artery is distorted by removal of the coronary arteries from the patching of the defects (in the case of the aorta), and the reimplantation of those arteries in the pulmonary (neo-aortic) root. Dilatation of the reconstructed neo-aortic root/ascending aorta is common following the arterial switch procedure, but clinically important insufficiency of the pulmonary valve-in the aortic position-is surprisingly rare: it occurs in less than $5 \%$. Images of the dilated ascending aortic aneurysms in ASO patients are often quite striking, but no cases of rupture or dissection have been reported. Data from the Children's Hospital of Boston show that marked dilatation of the aortic root is present in $95 \%$ of patients at 17 to 18 years of age, but most of the neo-aortic valves are competent, even in the presence of severe neo-aortic root dilatation. $^{6}$ 


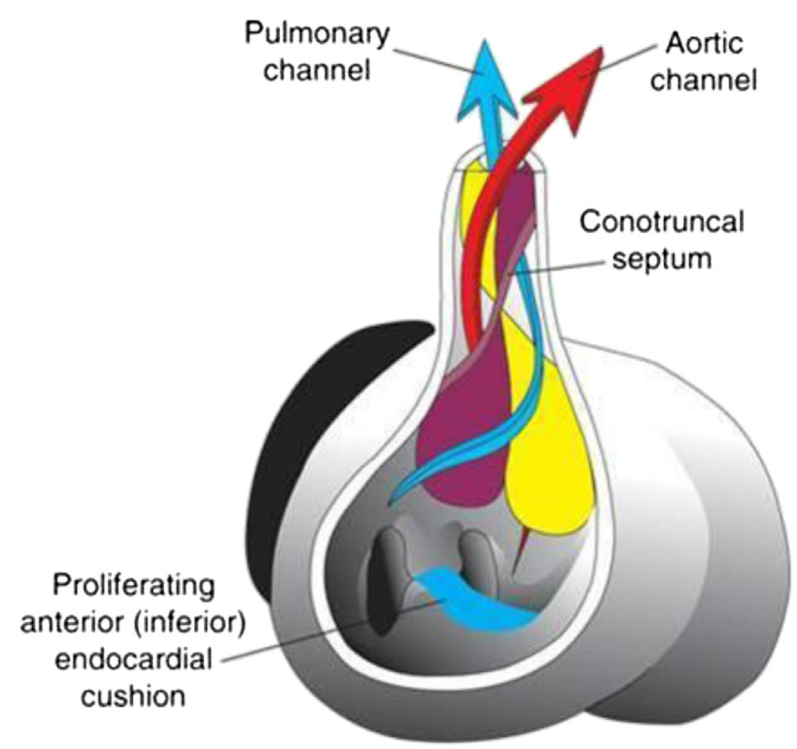

FIGURE 1. Normal septation of the conotruncus in heart development. Reprinted from https://www.studyblue.com.

The aorta also has a tendency to become aneurysmal following the Norwood procedure for repair of HLHS, one of the most severe congenital heart conditions, in which the left-sided structures of the heart fail to develop in utero. In HLHS, the aortic valve is small; the ascending aorta may be only 3 to $4 \mathrm{~mm}$ in diameter, and the aortic arch is hypoplastic. With the Norwood palliation, the neo-aorta is reconstructed using a portion of the proximal pulmonary artery, a strip of native aorta, and prosthetic materialusually cryopreserved homograft-so that there is unobstructed flow from the right ventricle and reliable, controlled pulmonary flow is provided either directly through a small conduit from the right ventricle to the pulmonary artery confluence (a "Sano" shunt), or using a modified Blalock-Taussig shunt from the subclavian artery to the pulmonary artery. The neo-aorta tends to dilate with time, often reaching quite large diameters. In this population, there is particular concern about the aneurysm pressing on pulmonary arteries and causing obstruction to pulmonary flow. Experience from the Children's Hospital of Philadelphia shows that virtually all neo-aortas become massively dilated, with $z$ scores of 5 or so after 5 to 10 years of follow-up. ${ }^{7}$ Nevertheless, there are no reported cases of rupture or dissection of these aortas, which should temper our enthusiasm for prophylactic intervention in the absence of pulmonary artery compression or neo-aortic valve incompetence.

There is also a well-known tendency of the aorta to dilate after the Ross procedure, both the transposed autograft pulmonary root and the native distal ascending aorta, particularly when the aortic valve is bicuspid. The paucity of reported cases of either autograft root dissection (1 case)
TABLE 1. Risk of aortic rupture and/or dissection by age and diagnosis

\begin{tabular}{lcccc}
\hline Diagnosis & Infancy & Childhood & Adolescence & Adulthood \\
\hline BAV & - & $+/-$ & + & + \\
Marfan & $+/-$ & $+/-$ & + & + \\
Loeys-Dietz & ++ & ++ & ++ & ++ \\
Postsurgical & - & - & + & + \\
\hline
\end{tabular}

$B A V$, Bicuspid aortic valve.

or rupture (none), suggest that a conservative approach is prudent, and that the traditional criteria for replacement of dilated ascending aorta and roots may not be applicable after the Ross procedure. When reintervention is necessary, it is usually for autograft valvar regurgitation; because the autograft leaflets are often normal or minimally distorted, these patients may be good candidates for valve-sparing procedures. The risk of aortic dilatation appears to be increased in patients who undergo operation at a young age, those with aneurysmal disease at the first operation, those with aortic regurgitation and BAV, and finally those who undergo full aortic root replacement with the pulmonary autograft, rather than insertion of the pulmonary valve within the native aorta. ${ }^{8}$

\section{Overall Risk of Rupture or Dissection in Patients With Congenital Problems: Loeys Dietz and Turner Syndromes}

In general, the risk of rupture or dissection in the first 2 decades of life is relatively low: it is extremely rare in childhood except in Loeys-Dietz and Turner syndromes. BAV and Marfan syndrome generally pose a problem only in late adolescence and adulthood. Although there are reports of rupture and dissection during the first 12 years of life, these aortic catastrophes are extremely rare. Table 1 summarizes the age- and diagnosis-related risk for rupture and/or dissection in patients with the most common forms of congenital problems of the aorta.

Loeys-Dietz syndrome is an exception to the generalization that aortopathies generally do not cause catastrophes in children. ${ }^{9}$ Indeed, rupture and dissection can occur at very young ages (6 months of age) and at very small (nearly normal) aortic diameters. This group of patients thus probably deserves a more aggressive surgical prophylactic approach.

The other group at risk in childhood is the cohort with Turner syndrome (gonadal dysgenesis, webbed neck, short stature, BAV, and coarctation), who have an increased risk of dissection. A recent study at the National Institutes of Health of more than 200 patients identified 3 patients who had an aortic dissection: all had aortic dilatation significantly greater than the overall group as a whole, but the diameter of the aorta at dissection was less than $5 \mathrm{~cm} .{ }^{10}$ In this group of patients, indexing the aneurysm size to body size may be important. 


\section{$Z$ SCORES}

A word of caution is appropriate about use of $z$ scores in the management of dilated aorta. The $z$ score describes the number of standard deviations from the mean of a given measurement when compared with normal individuals with the same body surface area, and so it provides a basis for comparison with other children of different body size. At the tail end of the distribution-the 95th to 97th percentile - a very small change in the measurement of the aorta can trigger a very large jump in the $z$ score. In these instances, it is important to maintain some perspective and to keep in mind the actual measurement of the aorta: the change in the $z$ score may be a consequence of a difference that is within the range of error of the aortic measurement.

In summary, there is a substantial and increasing number of patients with congenital heart disease who have undergone corrective surgery and now have large and progressively dilating aortas whose "natural" history is incompletely understood. The indications for surgery in this group of patients are therefore not yet evidence based. My plea is that we approach these patients judiciously and with caution and not replace every dilated aorta, particularly in patients who have had multiple sternotomies, aortopulmonary collaterals, and hearts enlarged from chronic volume and pressure overload. For them, surgery is an intimidating prospect. However, special consideration should be given to patients with Loeys-Dietz syndrome because of its particularly aggressive aortopathy, and to those with Turner syndrome, in which we know that body size does matter. In both Loeys-Dietz and Turner syndromes, rupture and dissection do occur at relatively small aortic diameters early in life.

\section{References}

1. Zanotti G, Vricella LA, Cameron DEC. Thoracic aortic aneurysm syndromes in children. Semin Thorac Cardiovasc Surg Pediatr Card Surg Ann. 2008;11:11-21.

2. Cramer JW, Ginde S, Bartz PJ, Tweddell JS, Litwin SB, Earing MG. Aortic aneurysms remain a significant source of morbidity and mortality after use of Dacron patch aortoplasty to repair coarctation of the aorta: results from a single center. Pediatr Cardiol. 2013:34:296-301.

3. Warnes CA, Williams RG, Bashore TM, Child JS, Connolly HM, Dearani JA, et al. ACC/AHA 2008 guidelines for the management of adults with congenital heart disease: a report of the American College of Cardiology/American Heart Association Task Force on Practice Guidelines. J Am Coll Cardiol. 2008;52: 143-263.

4. Niwa K, Perloff JK, Bhuta SM, Laks H, Drinkwater DC, Child JS, et al. Structural abnormalities of great arterial walls in the congenital heart disease. Light and electron microscopic analyses. Circulation. 2001;103:393-400.

5. Niwa K. Aortic root dilatation in tetralogy of Fallot long-term after repairhistology of the aorta in tetralogy of Fallot: evidence of intrinsic aortopathy. Int J Cardiol. 2005;103:117-9.

6. Schwartz ML, Gauvreau K, del Nido P, Mayer JE, Colan SD. Long-term predictors of aortic root dilation and aortic regurgitation after arterial switch operation. Circulation. 2004;110:128-32.

7. Cohen MS, Marino BS, McElhinney DB, Robbers-Visser D, van der Woerd W, Gaynor JW, et al. Neo-aortic root dilation and valve regurgitation up to 21 years after staged reconstruction for hypoplastic left heart syndrome. J Am Coll Cardiol. 2003;42:533-40.

8. Cameron DE, Vricella LA. What is the proper place of the Ross procedure in our modern armamentarium? Curr Cardiol Rep. 2007;9:93-8.

9. MacCarrick G, Black JH, Bowdin S, El-Hamamsy I, Frischmeyer-Guerrerio PA, Guerrerio AL, et al. Loeys-Dietz syndrome: a primer for diagnosis and management. Genet Med. 2014;16:576-87.

10. Matura LA, Ho VB, Rosing DR, Bondy CA. Aortic dilatation and dissection in Turner syndrome. Circulation. 2007;116:1663-70. 